\title{
Numerical modelling of desiccation cracking of clayey soil
}

\author{
Thi Dong Vo ${ }^{1}$, Ahmad Pouya ${ }^{2}$, Sahar Hemmati ${ }^{1}$ and Anh Minh Tang ${ }^{2}$ \\ ${ }^{1}$ IFSTTAR, Marne-la-Vallée, France \\ ${ }^{2}$ Ecole des Ponts ParisTech, Marne-la-Vallée, France
}

\begin{abstract}
The formation and propagation of desiccation cracks in soil is an extremely complex phenomenon because of the coupling between hydraulic and mechanical behaviour of soil, which are constituted here by the presence of capillary forces and discontinuities. The formation of a cracks network strongly influences the mechanical and hydraulic properties of soil. The main objective of this research is to study the evolution of suction and strain fields, the initiation and propagation of cracks under the effect of drying, using the finite element method. A simulation of a soil sample with four cohesive joints shows the results similar to experimental data. In addition, a simulation of multijoints shows that cracks does not open in all potentials positions and it gives similar spacing.
\end{abstract}

\section{Introduction}

The desiccation cracking is a common phenomenon in soils and rocks. The essential mechanisms of initiation and propagation of desiccation cracking are not yet well understood, and consequently, the method to predict, control or avoid cracking is inadequately developed. In soils, especially in clayey soils, the water content decreases due to evaporation. This reduction of water content is accompanied with increase of suction and effective stress which causes shrinkage. If the shrinkage is restrained (by frictional boundary conditions, concentration of stress or gradient of humidity...), it will generate a tensile stress. When this tensile stress reaches the tensile strength of the material, a crack network can be formed. The desiccation cracks increase infiltration of fluid and influence the transport of pollutions [1][2] . They affect also the performance of coverage of waste disposal and decrease the strength of earth embankments [3][4].

The formation and evolution of desiccation cracks have been studied by many authors experimentally and numerically. Some numerical simulations have been carried out by discrete element method and others by finite difference method. The objective of this research is to study the evolution of suction and strain fields during desiccation, as well as the propagation of desiccation cracks with taking into account the interaction between them. The simulation is carried out by using the finite element (FEM) 2D code Porofis [5].This code allows modelling hydro-mechanical coupling and fluid mass exchanges between fracture/matrix, fracture/fracture, and fracture/boundary. The hydro-mechanical coupling in this code is realized by an iterative process passing from the hydraulic to the mechanical problem and vice versa (weak numerical coupling). In addition, the cohesive

a Corresponding author: thi-dong.vo@cermes.enpc.fr fracture law [6] allows simulating crack propagation under drying process. Two examples of simulation with four cohesive joints and multi-joints will be presented in the following in order to illustrate first the soil surface settlement and then initiation and propagation of desiccation cracks.

\section{Model descriptions}

A heterogeneous porous body $\Omega$ containing a set of joints is considered. Both the porous matrix and joints are subjected to fluid flow and deformation (Figure 1)
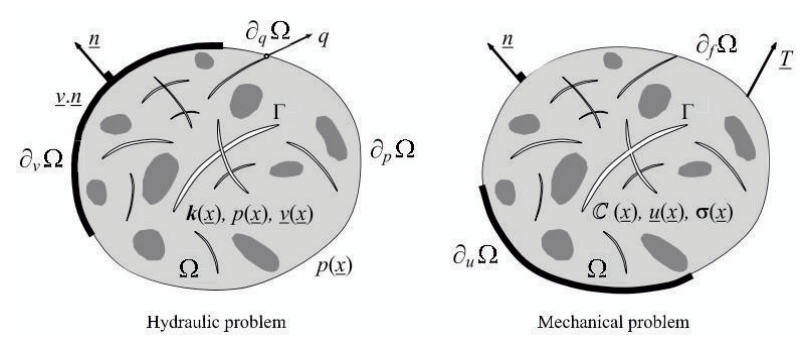

Figure 1. Hydraulic and mechanical problems

\subsection{Hydraulic diffusion around the crack}

The flow in the soil around the crack is governed by the Darcy's law:

$$
\underline{v}=-k \nabla p
$$

with $k=k_{m} / \mu$ where $k_{m} \quad\left(\mathrm{~m}^{2}\right)$ is the intrinsic permeability, $p(\mathrm{MPa})$ the pore pressure and $\mu$ (Pa.s) the fluid dynamic viscosity. In absence of source term, 
for an unsaturated medium the balance of fluid mass becomes:

$$
\frac{d\left(\rho_{f} \phi S\right)}{d t}-\operatorname{div}\left(\rho_{f} k \nabla p\right)=0
$$

Here, the fluid mass $m_{f}$ is calculated in unit volume and $m_{f}=\rho_{f} \phi S$ where $\phi$ is the porosity, $\rho_{f}$ is the fluid density and $S$ is the degree of saturation of medium. To make possible a standard formulation for simulation by FEM, the variation of $m_{f}$ is written as a function of the nodal pressure $p$ as follows:

$$
\frac{d m_{f}}{m_{f}}=\frac{d \rho_{f}}{\rho_{f}}+\frac{d \phi}{\phi}+\frac{d S}{S}
$$

The variation of fluid density $\rho_{f}$ is related to the pressure by the following relation with $K_{f}$ is the fluid compressibility.

$$
\frac{d \rho_{f}}{\rho_{f}}=\frac{d p}{K_{f}}
$$

The variation of porosity $\phi$ is related to the pressure by using the poromechanical model:

$$
d \phi=b d \varepsilon_{v}+\frac{d p}{N}
$$

with $\mathrm{b}$ the Biot's coefficient and $\mathrm{N}$ the Biot modulus. Theses parameters are linked with the bulk modulus of skeleton solid grain, $\mathrm{K}_{\mathrm{s}}$, by the following relationship:

$$
b=1-K / K_{s} \quad 1 / N=\left(b-\phi_{0}\right) / K_{s}
$$

In reality, the increase of suction is accompanied with increase of bulk modulus and the tensile strength of soil. But in the present work, a constant Young modulus is assumed.

During drying, the degree of saturation decreases. This value is related to suction by the water retention curve :

$$
\frac{S-S_{r}}{1-S_{r}}=\frac{1}{\left(1+(\alpha p)^{n}\right)^{m}}
$$

In this equation $\alpha, n$ are the coefficients, $m=1-1 / n$ and $S_{r}$ is the residual degree of saturation of soil. In this work, $S_{r}$ assumed equal to $2 \%$. From Eq.(7), we can deduct:

$$
\frac{d S}{S}=\frac{d S}{d p} \frac{d p}{S}=\left(1-S_{r}\right)\left\{-m \cdot\left[1+(\alpha p)^{n}\right]^{-m-1} \cdot\left(\alpha^{n} n p^{n-1}\right)\right\} \frac{d p}{S}
$$

During drying, the change of permeability is considered as a function of its permeability in the saturated state and the degree of saturation. The following expression is adopted for this study:

$$
k=k_{s}\left(\frac{S-S_{r}}{1-S_{r}}\right)^{3}
$$

Replacing Eq. (3),(4), (5), (8) in Eq. (2), we found the final equation for hydraulic problem:

$$
C_{M} \frac{d p}{d t}+S b \frac{d \varepsilon_{v}}{d t}-\operatorname{div}(k \nabla p)=0
$$

with $C_{M}=S\left(\frac{\phi}{K_{f}}+\frac{1}{N}+\frac{\phi^{d S} / d p}{S}\right)$. In this work, the effect of volumetric strain of solid skeleton to the hydraulic diffusion, i.e. the term $S b \frac{d \varepsilon_{v}}{d t}$ is neglected. The Eq.(10) after this simplification becomes:

$$
C_{M} \frac{d p}{d t}=\operatorname{div}(k \nabla p)
$$

\subsection{Fluid flow in the crack}

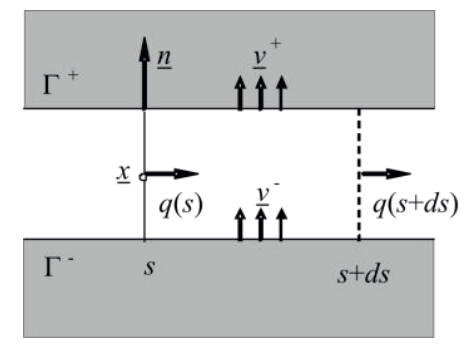

Figure 2. Masse exchange between crack and matrix [7]

We assumed that the cross conductivity of crack is infinite which imply that the pressure is the same on two sides of crack and maybe presented by only value $p$ that is variable along the crack. The flow rate $q$ in the crack is governed by the cubic law:

$$
q(s)=-c(s) \partial_{s} p
$$

where $c_{f}$ is the conductivity tensor in the crack plane and $s$ presents curvilinear abscise along the crack. In the most cases $c_{f}$ is supposed isotropic in the tangent plane and represents by a scalar $c$. This hydraulic conductivity can be related to crack opening $e$ by the Poiseuille's law $c=e^{3} / 12 \mu$. Flow in crack taking into account the fluid exchange between crack and matrix, crack and 
crack, crack and boundary is usually a difficulty of modelling of cracking. In most of the researches, this problem isn't considered. The balance of fluid mass in the crack taking into account the mass exchange with surrounding matrix is written as:

$$
\partial_{s}\left(\rho_{f} q\right)+\left\|\rho_{f} \underline{v}\right\| \cdot \underline{n}+e \frac{d \rho_{f}}{d t}=0
$$

where $\|\underline{v}\|$ presents the fluid velocity discontinuity or the velocity jump between the two sides of the fracture, and $\underline{n}$ is the unit normal to the fracture surface (Figure 2). We neglected the change of thickness, i.e. term $\partial e / \partial t$, in solving the Eq.(13), but this opening cracks $e$ is accounted in updating the hydraulic conductivity of cracks (Poiseuille's law). Also the final equation is obtained by replacing Eq. (12) in Eq.(13) and in using the compressibility of fluid:

$$
C_{F} \frac{d p}{d t}=\partial_{s}\left(c \partial_{s} p\right)-\|\underline{v}\| \cdot \underline{n}
$$

with $C_{F}=e / K_{f}$

\subsection{Mechanical behaviour}

In this work, the soil is assumed to have a poroelastic linear behaviour reads:

$$
\varepsilon_{i j}=\frac{1+v}{E} \sigma_{i j}-\frac{v}{E} \sigma_{k k} \delta_{i j}+\frac{b}{3 K} p \delta_{i j}
$$

with $\varepsilon_{v}=\varepsilon_{k k}$ the volumetric strain and $K=E / 3(1-2 v)$ the drained bulk modulus.

In this model the joints are simulated as elements of zero thickness with a very small hydraulic conductivity and an important stiffness in the first. For the mechanical behaviour of joint elements, the cohesive fracture law [6] is applied. A damage variable $D$ is added in the model to present the process of damage through decrease of crack stiffness and evolution of load surface. Under the effect of evaporation, the tensile stress increases with suction and as soon as this tensile stress reaches the tensile strength, the crack initiates. In the code, the crack opening is calculated by the displacement discontinuity on the two sides of crack and represented by relative displacement vector $\underline{u}$. It is related to the stress by a crack stiffness tensor $k$ and the damage variable $D$ by the relationship: $\sigma=(1-D) k u$. The criterion of damage includes the evolution of mechanical strength as well as process of damage:

$$
F(\sigma, D)=\tau^{2}-\sigma_{n}^{2} \tan ^{2} \varphi+2 g(D) \sigma_{c} \sigma_{n}-g^{2}(D) C^{2}
$$

where $\tau=\sigma-\sigma_{n} n$. In this equation, $\mathrm{C}$ represents the cohesion of intact (undamaged) joint, $\varphi$ the friction angle and $\sigma_{c}$ an auxiliary constant parameter related to $\mathrm{C}$, $\varphi$ and the tensile strength $\sigma_{R}$ of intact joint by the following relation:

$$
\begin{gathered}
\sigma_{c}=\frac{C^{2}+\sigma_{R}^{2} \tan ^{2} \varphi}{2 \sigma_{R}} \\
g(D)=(1-D)(1-\beta \ln (1-D))
\end{gathered}
$$

The function $g(D)$ plays a key role in this constitutive model. It takes the value 1 for the initial intact state and zero for the ultimate failure or completely damaged state.

In this model, the coefficient $\beta$ presents the ductility of the material. The ductility of material increases with $\beta$. The damage evolution is calculated through the evolution of damage variable $\mathrm{D}$, by using the equation of associated consistence $d F=0$. The damage evolution law must ensure the positivity of energy dissipation in the damage process. The completed damage evolution is then following

$$
\begin{cases}d D=0 & \text { if } F<0 \\ d D=0 & \text { if } F=0 \text { and }(\partial F / \partial \sigma) \dot{\sigma}<0 \\ d D=-(\partial F / \partial D)^{-1}(\partial F / \partial \sigma) \dot{\sigma} \text { elsewhere }\end{cases}
$$

\subsection{Hydro-mechanical coupling}

Eq.(11) and Eq.(14) are the base of hydraulic simulation in the code Porofis. The suction which is a result in the hydraulic problem is introduced after in the mechanical model to find the stress and strain fields. The opening of cracks calculated in the mechanical problem plays the role to update the cracks hydraulic conductivity like Poiseuille's law. The hydro-mechanical coupling in the code is carried out as showing in the Figure 3:

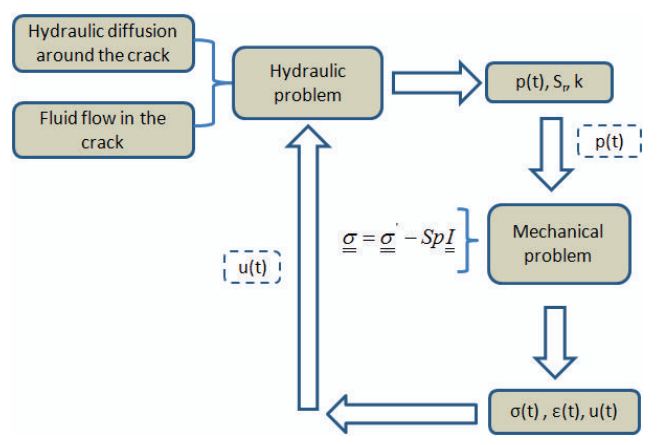

Figure 3 : Hydro-mechanical coupling in the code Porofis

\section{Numerical simulations}

\subsection{Description of test [8]}

Sanchez et al. [8] carried out a desiccation test in laboratory. A plate circular silt of $100 \mathrm{~mm}$ of diameter 
and $13 \mathrm{~mm}$ of thickness in slurry state is prepared and let in chamber condition with temperature $19.5^{\circ}\left( \pm 0.3^{\circ} \mathrm{C}\right)$ and relative humidity $37 \%( \pm 4 \%)$. By using the $2 \mathrm{D}$ profile laser technique, the characteristics soil in drying are observed (volume change, water loss...). Especially, the evolution crack network's morphology (thickness, depth) is observed in the vertical plane. The main results as settlement, degree of saturation and crack development are used to discus in this study of numerical simulation.

\subsection{Parameters determination}

The water retention curve is fitted with experimental data [9] on the same type of soil, but in the compacted state (see Figure 4). We kept the air-entry value and used the Van-Genuchten model to determine the curve. The distance of two curves presents the difference between slurry and a compacted soil. The coefficients in VanGenuchten model are found: $\alpha=9.81$ (1/MPa) and $n=1.60$

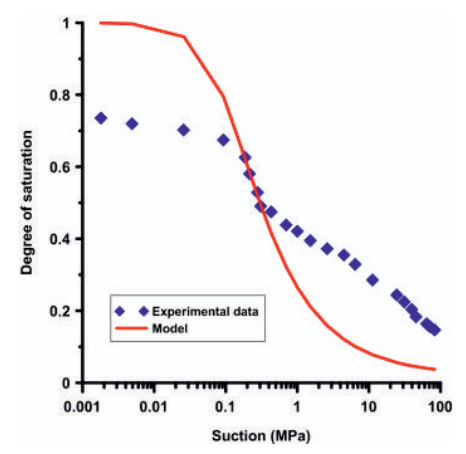

Figure 4 : Water retention curve

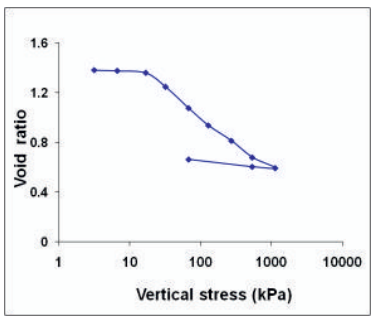

(a)

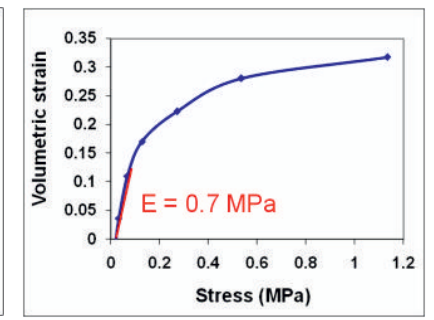

(b)
Figure 5 : (a) Odometer curve of compacted soil [10] and (b) Stress-volumetric strain curve

The soil is assumed linear elastic and isotropic with $v=0.3, \quad \phi=0.3, \quad K_{s}=30(G P a), \quad k=5 \times 10^{-11}$. $(\mathrm{m} / \mathrm{s})$. The elastic modulus is found from odometer curve [10] (Figure 5a). This odometer curve is translated to volumetric strain/stress curve (Figure 5 b). The modulus $\mathbf{E}$ $=0.7 \mathrm{MPa}$ is so deducted equal to the slope on the stressvolumetric strain curve.

The fluid characteristics are: $\rho_{f}=1000\left(\mathrm{~kg} / \mathrm{m}^{3}\right)$, $\mu=10^{-3}(\mathrm{~Pa} / \mathrm{s}), K_{f}=2.2(\mathrm{GPa})$
The parameters of joints are: $K_{t t}=1 \mathrm{MPa} / \mathrm{mm}$; $K_{n n}=700 \mathrm{MPa} / \mathrm{mm} ; K_{t n}=K_{n t}=0 \mathrm{MPa} / \mathrm{mm}$, $C=0.06 \mathrm{MPa}, \varphi=25^{\circ} ; \beta=1.5 ; e_{0}=0.0002 \mathrm{~mm}$.

\subsection{Numerical model}

The first simulation is a rectangle of $100 \mathrm{~mm} \times 13 \mathrm{~mm}$ (similar to experimental test) including four cohesive joints of $10 \mathrm{~mm}$ of length (from $\mathrm{Y}=3 \mathrm{~mm}$ to $\mathrm{Y}=13 \mathrm{~mm}$ ) in $X=5,15,50,80 \mathrm{~mm}$ (the same positions observed in the experimental test) under plane strain condition (Figure 6). This simulation allows observing two phases of desiccation and evolution of cracks opening.

For hydraulic boundary conditions, evaporation rate with condition of temperature $20^{\circ}$ and relative humidity of $40 \%$ is applied on the top surface sample. This rate evaporation will decrease with time, as a function to suction on the top surface like the relation proposed by Wilson, 1990 [11]. For mechanical boundary conditions, the horizontal and vertical displacements are blocked on the left, right and bottom edges to present a perfect cohesion between the soil sample and the mold.

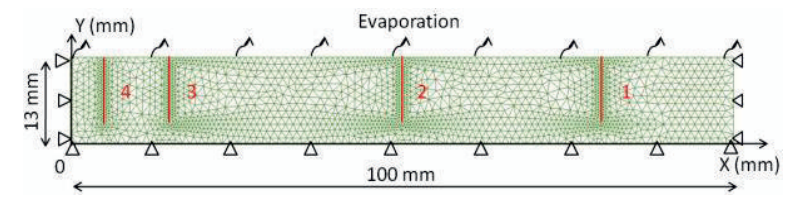

Figure 6 : Geometry and boundary conditions of simulation

\subsection{Results}

The Figure 7 presents profile of suction including the deformation evolution of the sample with time. We distinguish clearly two phases in the drying process. In the first phase $(\mathrm{t}=0$ to $\mathrm{t}=4 \mathrm{~h})$, the soil settlement increases, but all the joints remain close. We can imagine that in this time, there is only soil consolidation without any crack initiation. Then, at $t=4.2 \mathrm{~h}$, the first crack opened and during drying, the other cracks opened gradually.

The Figure 8 presents the suction profile in time for the section $X=5 \mathrm{~mm}$ (position of joint 4 ). Because the thickness of sample is very small $(13 \mathrm{~mm})$, we find also that from $\mathrm{t}=0$ to $\mathrm{t}=4 \mathrm{~h}$ (before onset of cracks), the suction gradient is almost homogenous and continuous from top to bottom. After the opening of this crack, this profile suction is devised in two part at $\mathrm{Y}=3 \mathrm{~mm}$, the limit between joint and soil matrix. Because as soon as the crack is opened, its hydraulic conductivity increases according to the Poiseuille's law. From this moment, the suction is the same and increases more quickly in the crack (10 mm of length from $\mathrm{Y}=3 \mathrm{~mm}$ to $\mathrm{Y}=13 \mathrm{~mm}$ ) than in the soil matrix (from $\mathrm{Y}=0 \mathrm{~mm}$ to $\mathrm{Y}=3 \mathrm{~mm}$ ). 


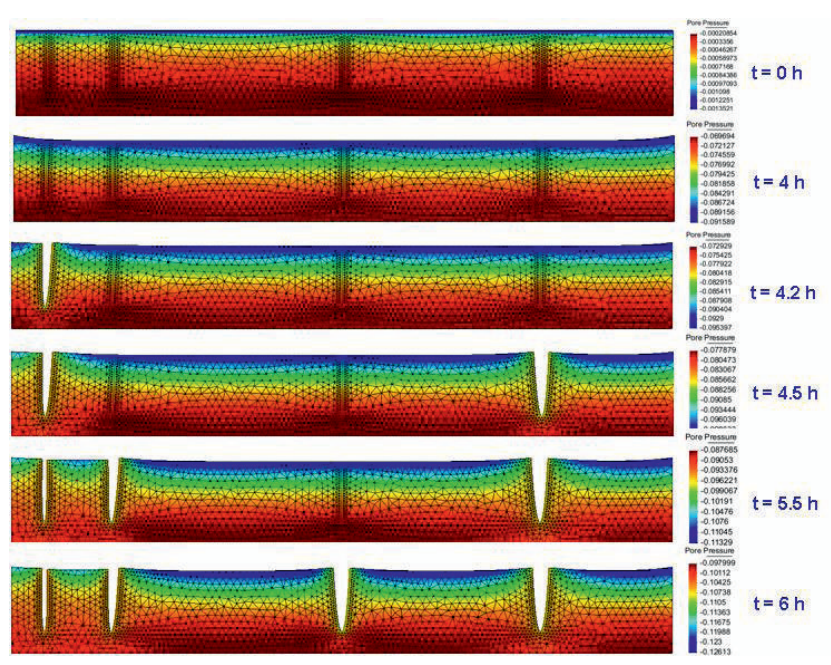

Figure 7 : Evolution of deformation sample in time

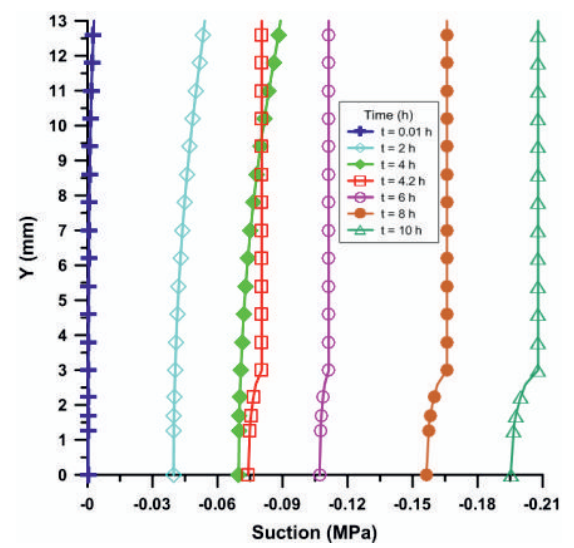

Figure 8 : Suction profile in the section $X=5 \mathrm{~mm}$

Evolution of settlement at the middle surface of sample $(\mathrm{X}=50 \mathrm{~mm}, \mathrm{Y}=13 \mathrm{~mm})$ is considered. The Figure 9 presents the comparison between this evolution in time for numerical model and experimental data [8]. It shows that there isn't the difference between the ones. Additionally, the deformable form before cracking ( $\mathrm{t}=4 \mathrm{~h}$ in Figure 7) is similar to the experimental observation.

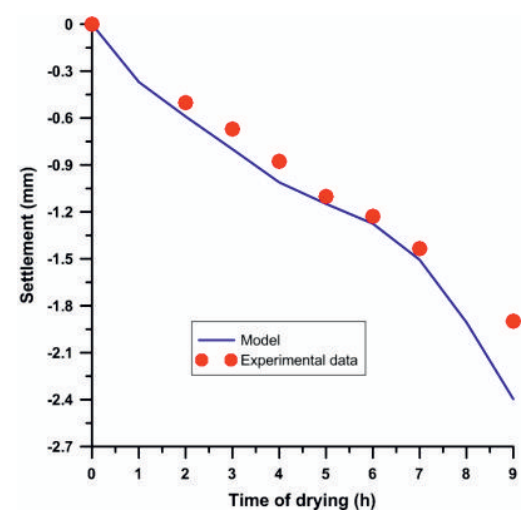

Figure 9 : Settlement in time for numerical simulation and experimental data [8]

In the FEM code, the opening of a crack is calculated as the displacement discontinuity of two sides of the crack. The Figure 10 presents the increase of opening for
4 cracks. Until $\mathrm{t}=4 \mathrm{~h}$, the opening of all cracks is zero, corresponding the consolidation phase without cracking and elastic behavior for joint elements. When the tensile strength was reached, the damage phase began and we obtained the displacement jump on opening cracks. The interaction between cracks 3 and 4 is displayed in the Figure 10 . We observe that the crack 4 is onset before crack 3 . But when the crack 3 is opened $(t=4.5 \mathrm{~h})$, the opening of crack 4 starts to be reduced, but the crack 1 and crack 2, far from the crack 4 , and the zone between them were not influenced.

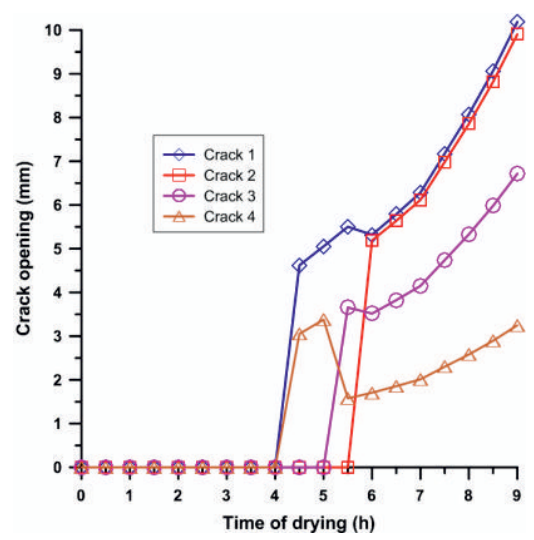

Figure 10 : Development of opening cracks in time

\subsection{Simulation with multi-joint elements}

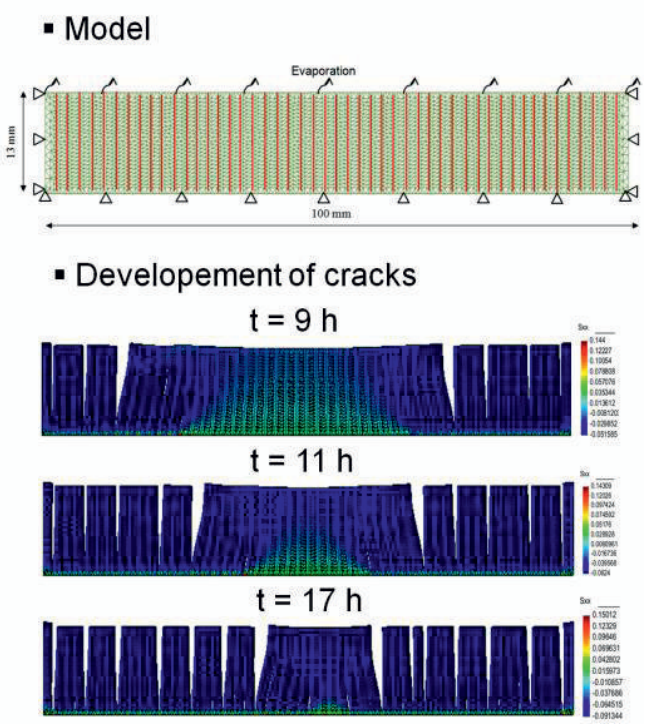

Figure 11 : Simulation with 50 joints

For further discussion, a preliminary result using 50 cohesive joints is showed in the Figure 11. In this simulation, 50 cohesive joints (one joint every $2 \mathrm{~mm}$ ) are set up to present the potential cracks (Figure 11). The result of this simulation showed that the crack doesn't onset on all positions or joints simultaneously. In this simulation, the cracks were generated with an average spacing of $6 \mathrm{~mm}$. In addition, this simulation showed clearly the interaction between cracks. The opening of a new crack induces the closure of other nearby cracks. 
This simulation shows also that the cohesive fracture law applied could allow simulating the desiccation cracking and estimating an average spacing between cracks that will be studied in the future work.

\section{Conclusions}

This work presents an approach for desiccation cracking simulation talking into account the hydro-mechanical coupling: flow and deformation in presence of cracks by using the FEM code Porofis. The simulation including the cohesive joints shows that this approach allows simulating two phases of desiccation process: the consolidation one without cracking and the cracks development one. In addition, the simulation allows observing the evolution of soil parameters during drying as: suction, degree of saturation, settlement and cracks opening. The comparison of the numerical simulation with the experimental data [8] allowed evaluating the model.

This work showed that the cohesive fracture model is a good tool to simulate the evolution of desiccation cracks. Study of spacing and depth of cracks during drying will be investigated in future work in order to better understand the mechanisms of desiccation cracking.

\section{Acknowledgements}

The first author would like to thank to the COST Action TU1202 for fruitful exchange during the Workshop "Vadose Zone Workshop" from $5^{\text {th }}$ July 2015 to $8^{\text {th }}$ July 2015 that have been useful for this study.

\section{References}

[1] E. Drumm, D. Boles, and G. Wilson, "Desiccation cracks result in preferential flow," Environ. Geotech., pp. 22-26, 1997.

[2] M. Flury, H. Flühler, W. A. Jury, and J. Leuenberger, "Susceptibility of soils to preferential flow of water: A field study," Water Resour. Res., vol. 30, no. 7, pp. 1945-1954, 1994.

[3] W. H. Albright, C. H. Benson, G. W. Gee, T. Abichou, S. W. Tyler, and S. a. Rock, "Field performance of a compacted clay langfill final cover at a humid site," J. Geotech.
Geoenvironmental Eng., vol. 132, pp. 13931403, 2006.

[4] P. D. Jackson, K. J. Northmore, P. I. Meldrum, D. a. Gunn, J. R. Hallam, J. Wambura, B. Wangusi, and G. Ogutu, "Non-invasive moisture monitoring within an earth embankment - A precursor to failure," NDT E Int., vol. 35, pp. 107-115, 2002.

[5] A. Pouya, "A finite element method for medeling coupled flow and deformation in porous fractured media," Int. J. Numer. Anal. Methods Geomech., vol. 32, 2015.

[6] A. Pouya and P. Bemani, "A damage-plasticity model for cohesive fractures," Int. J. Rock Mech. Min. Sci., vol. 73, pp. 194-202, 2015.

[7] A. Pouya and S. Ghabezloo, "Flow Around a Crack in a Porous Matrix and Related Problems," Transp. Porous Media, vol. 84, pp. 511-532, 2010 .

[8] M. Sanchez, A. Atique, S. Kim, E. Romero, and M. Zielinski, "Exploring desiccation cracks in soils using a 2D profile laser device," Acta Geotech., vol. 8, no. 6, pp. 583-596, Oct. 2013.

[9] G. El Mountassir, M. Sánchez, E. Romero, and R. a a Soemitro, "Behaviour of compacted silt used to construct flood embankment," Proc. ICE Geotech. Eng., vol. 164, no. 3, pp. 195-210, Jun. 2011.

[10] G. El Mountassir, M. Sánchez, and E. Romero, "An experimental study on the compaction and collapsible behaviour of a flood defence embankment fill," Eng. Geol., vol. 179, pp. 132145, Sep. 2014.

[11] G. W. Wilson, "Soil Evaporative Fluxes for Geotechnical Engineering Problems,” 1990. 